OPEN ACCESS

Edited by:

Khosrow Kashfi,

City University of New York,

United States

Reviewed by:

Manish Sharma,

Emory University, United States

Huabo Su,

Augusta University, United States

*Correspondence:

Nuo Sun

nuo.sun@osumc.edu

Specialty section:

This article was submitted to Pharmacology of Anti-Cancer Drugs,

a section of the journal

Frontiers in Pharmacology

Received: 16 November 2021

Accepted: 24 January 2022

Published: 17 February 2022

Citation:

Zhang R, Ozgen S, Luo H, Krigman J, Zhao Y, Xin G and Sun N (2022) The Mitochondrial Deubiquitinase USP3O

Regulates AKT/mTOR Signaling.

Front. Pharmacol. 13:816551.

doi: 10.3389/fphar.2022.816551

\section{The Mitochondrial Deubiquitinase USP30 Regulates AKT/mTOR Signaling}

\author{
Ruohan Zhang ${ }^{1,2}$, Serra Ozgen ${ }^{1}$, Hongke Luo ${ }^{1}$, Judith Krigman ${ }^{1}$, Yutong Zhao ${ }^{1}$, Gang Xin ${ }^{3}$ \\ and Nuo Sun ${ }^{1 *}$ \\ ${ }^{1}$ Department of Physiology and Cell Biology, The Ohio State University Wexner Medical Center, Columbus, OH, United States, \\ ${ }^{2}$ Division of Pharmaceutics and Pharmacology, The Ohio State University College of Pharmacy, Columbus, OH, United States, \\ ${ }^{3}$ Pelotonia Institute for Immuno-Oncology, The Ohio State University Comprehensive Cancer Center, Columbus, OH, \\ United States
}

Mitophagy is an intracellular mechanism to maintain mitochondrial health by removing dysfunctional mitochondria. The E3 ligase Parkin ubiquitinates the membrane proteins on targeted mitochondria to initiate mitophagy, whereas USP30 antagonizes Parkindependent mitophagy by removing ubiquitin from Parkin substrates. The AKT/mTOR signaling is a master regulator of cell proliferation, differentiation, apoptosis, and autophagy. Although mounting evidence suggests that perturbations in the AKT/ mTOR signaling pathway may contribute to mitophagy regulation, the specific mechanisms between Parkin/USP30 and AKT/mTOR signaling have not been elucidated. In this study, we employ a set of genetic reagents to investigate the role of Parkin and USP3O in regulating the AKT/mTOR signaling during mitophagy. We demonstrated that, in the setting of mitochondrial stress, the AKT/mTOR signaling is regulated, at least in part, by the activity of Parkin and USP30. Parkin inhibits AKT/ mTOR signaling following an in vitro mitochondrial stress, thereby promoting apoptosis. However, USP30 overexpression antagonizes the activity of Parkin to sustain AKT/mTOR activity and inhibit apoptosis. These findings provide new insights into Parkin and USP30's role in apoptosis and suggest that inhibiting USP30 might provide a specific strategy to synergize with AKT/mTOR inhibitors in cancer treatment.

Keywords: USP30, parkin, mitophagy, akt, mTOR, leukemia, cancer

\section{INTRODUCTION}

Mitophagy, also known as mitochondria-specific autophagy, is an evolutionarily conserved cellular mechanism to recycle specific mitochondria, by being encapsulated by the structurally doublemembrane autophagosome (Youle and Narendra, 2011; Dikic and Elazar, 2018; Saha et al., 2018; Khandia et al., 2019). Mitophagy plays an essential role in maintaining mitochondrial health and metabolic reactions during cellular stress, such as hypoxia or starvation (Palikaras et al., 2018; Killackey et al., 2020).

Mitophagy is highly regulated (Chu, 2011). The PTEN (Phosphatase and tensin homolog)induced kinase 1 (PINK1) and the cytosolic E3 ubiquitin ligase Parkin are important mitophagy promoters (Narendra et al., 2010; Ding and Yin, 2012; Ge et al., 2020). When mitochondria are 
damaged, PINK1 accumulates on the outer mitochondrial membrane (OMM) and recruits Parkin from the cytosol to ubiquitinate mitochondrial membrane proteins, including the translocase of the outer membrane subunit 20 (TOM20) (Narendra et al., 2010; Yoshii et al., 2011; Lazarou et al., 2015; Bingol and Sheng, 2016; Palikaras et al., 2018; Killackey et al., 2020). Ubiquitin chains on the mitochondrial membrane tag the mitochondria and induce the organelle's engulfment by the autophagosome (Narendra et al., 2010; Ding and Yin, 2012; Lazarou et al., 2015; Bingol and Sheng, 2016; Palikaras et al., 2018). Ubiquitin carboxylterminal hydrolase 30 (USP30) works as an essential checkpoint for mitophagy initiation (Liang et al., 2015; Kluge et al., 2018; Luo et al., 2021). USP30 is an OMM deubiquitinase that cleaves the Parkin-mediated ubiquitin chains to inhibit mitophagy (Liang et al., 2015; Bingol and Sheng, 2016). Expressing Parkin or inhibiting USP30 can promote mitophagy, improve mitochondrial functions, and rescue the symptoms of certain mitochondrial-related diseases (Bingol et al., 2014; Krishnmurthy and Shradgan, 2014). However, it remains possible that cells may become more vulnerable to specific mitochondrial stresses, and cell death may occur if the mitophagy response is too exuberant (Carroll et al., 2014; Liang et al., 2015; Wanderoy et al., 2020).

Another significant mitophagy regulator is the AKT (Protein kinase B)/mTOR (The mechanistic target of rapamycin) signaling (Kim and Guan, 2015; Soutar et al., 2018; de la Cruz López et al., 2019). The AKT/mTOR signaling is an intracellular pathway that plays a vital role in regulating cell survival (Manning and Toker, 2017; Xu et al., 2020). Previous studies indicate that the AKT/mTOR signaling inhibits mitophagy and promotes cell survival under mitochondrial stress (Akundi et al., 2012; Yang et al., 2017; Soutar et al., 2018; de la Cruz López et al., 2019; Wanderoy et al., 2020). However, it remains unclear how Parkin/USP30 and the AKT/mTOR signaling interact. Of note, during mitochondrial stress, Parkin expression or USP30 inhibition may induce cell apoptosis (Carroll et al., 2014; Liang et al., 2015). Interestingly, the AKT/mTOR pathway is dysregulated and hyperactive in $50-80 \%$ of human leukemia cases (Park et al., 2010; Nepstad et al., 2020). AKT hyperactivation correlates with aggressive cancer progression and resistance to a plethora of chemotherapeutics (Arafeh and Samuels, 2019). While targeting The AKT/mTOR pathway could serve as promising strategies for cancer treatment, the efficacy of monotherapy with AKT inhibitors is limited (Fransecky et al., 2015; Estruch et al., 2021). Further investigation of USP30's function in regulating AKT/mTOR signaling may offer new therapeutic approaches in cancer treatment.

In our study, Hela cells engineered to express Parkin (Hela Parkin cells) were exposed to a cocktail of mitochondrial inhibitors (antimycin plus oligomycin; AO). Consistent with previous observations, the addition of $\mathrm{AO}$ triggered rapid PINK1/Parkin mediated-mitophagy in vitro (Narendra et al., 2010; Vives-Bauza et al., 2010; Lazarou et al., 2015; Ordureau et al., 2018). In addition, we observed Parkin-dependent AKT downregulation and increased cell apoptosis after AO treatment. In this context, the increased expression of USP30 prevented $\mathrm{AKT}$ inactivation in response to $\mathrm{AO}$ treatment. Moreover, inhibiting USP30 decreased AKT levels in Hela Parkin USP30 cells and Jurkat T leukemia cells during mitochondrial stress and chemotherapies, theraby inducing cell apoptosis. Furthermore, We performed a chemical screening, suggesting that USP30 inhibitors may synergize with AKT/mTOR inhibitors in treating leukemia. Taken together, we demonstrated that Parkin and USP30 might regulate the AKT/mTOR signaling and cell survival during mitophagy, suggesting USP30 may serve as a potential drug target for leukemia treatment.

\section{RESULT}

\section{Parkin and USP30 Regulate Mitophagy Independent Cell Apoptosis in Response to Mitochondrial Stress}

We treated Hela Parkin cells and Hela Parkin USP30 cells with a cocktail of the mitochondrial complex III inhibitor Antimycin A and the ATP synthase inhibitor Oligomycin (AO) for up to $9 \mathrm{~h}$ (Baudot et al., 2015; Zachari et al., 2019). NDP52 and OPTN are adaptor proteins that link ubiquitinated mitochondria to the autophagosome. These two proteins are degraded along with the mitochondria during mitophagy (Lazarou et al., 2015; Zachari et al., 2019). As expected, Parkin induced rapid mitophagy during AO treatment, shown as the degradation of TOM20, NDP52, and OPTN in Figure 1, a. PARP is a universal protein cleaved only during apoptosis (Gobeil et al., 2001; D’Amours et al., 2001). In the presence of Parkin, AO treatment resulted in an increase of cleaved PARP, suggesting an increase in cell apoptosis (Figure 1A). Consistent with the western blot results, our cell viability tests (by resazurin assay) indicated substantial cell death after $24 \mathrm{~h}$ of AO treatment (Figure 1B). The decrease in AKT and cell apoptosis required Parkin to be activated because knocking out PINK1, Parkin'activator, abolished mitophagy and cell apoptosis (Figures 1B,C). In Hela Parkin USP30 cells, we observed increased USP30 protein levels compared to Hela and Hela Parkin cells (Figure 1A and Supplementary Figure 1A). In addition, USP30 overexpression prevented mitophagy and reduced cell apoptosis following AO treatment (Figures 1A,D). By treating cells with $10 \mathrm{ug} / \mathrm{ml}$ ST-539, a specific USP30 inhibitor (Luo et al., 2021), Parkin mediated mitophagy, and cell apoptosis resumed (Figures 1D,E). We next asked whether the Parkin/USP30-mediated cell apoptosis during mitochondrial stress is mitophagy dependent. AutophagyRelated-Gene 5 (ATG5) is essential for autophagosome formation. Knocking out ATG5 undermines autophagy and mitophagy (Mai et al., 2012; Ye et al., 2018; Zheng et al., 2019). Cell viability data showed that knocking out ATG5 did not limit cell death during mitochondrial stress (Figure 1F), suggesting the Parkin/USP30-regulated cell apoptosis during mitochondrial stress is mitophagy independent. To summarize, Parkin promotes apoptosis, while USP30 antagonizes mitophagyindependent cell apoptosis during mitochondrial stress. 


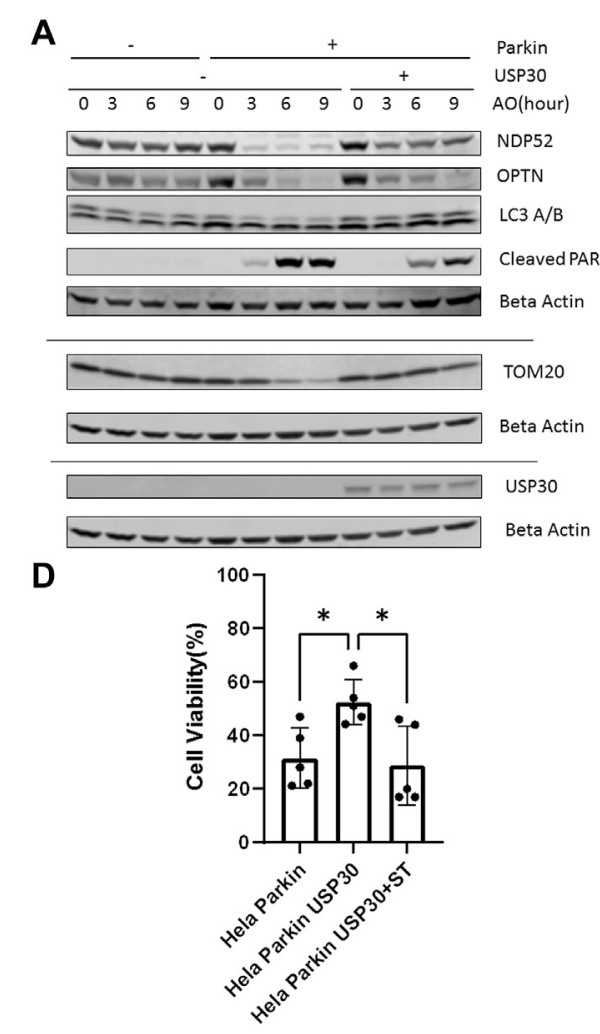

B

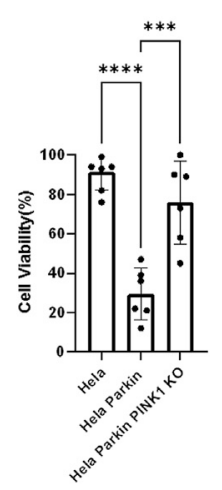

E

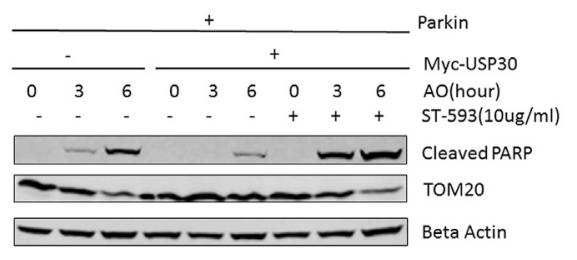

C

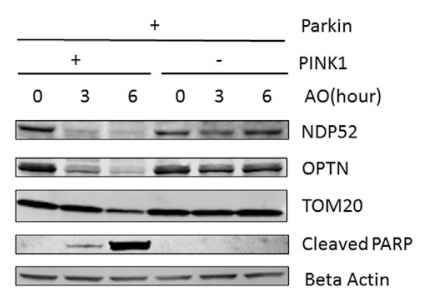

$\mathbf{F}$

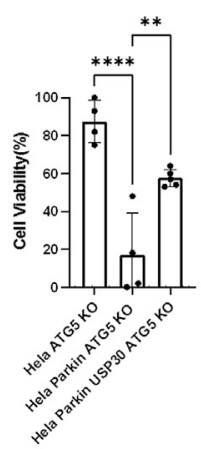

FIGURE 1 | PINK1/Parkin-dependent mitophagy induces apoptosis during mitochondrial stress (A). Western blot analysis of mitophagy proteins and the proapoptosis signal in Hela (no Parkin) cells and Hela Parkin cells after the AO (Antimycin A+ oligomycin) treatment. Cells were treated with AO at $5 \mathrm{ug} / \mathrm{ml}$ for $0,3,6$, and $9 \mathrm{~h}$. Beta Actin served as the loading control. These are representative figures from three independent experiments (B). Cell viability assay of Hela (no Parkin) cells and Hela Parkin cells after $\mathrm{AO}$ treatment. Cells were treated with $\mathrm{AO}$ at $5 \mathrm{ug} / \mathrm{ml}$ for $24 \mathrm{~h}$ and then incubated with resazurin for $2 \mathrm{~h}$. Fluorescence was read using $544 \mathrm{~nm}$ excitation and $590 \mathrm{~nm}$ emission wavelength. It is a representative figure from three independent experiments (C). Western blot analysis of mitophagy proteins and the pro-apoptosis signal in Hela Parkin cells and Hela Parkin with PINK1 KO cells after the AO treatment. Cells were treated with AO at $5 \mathrm{ug} / \mathrm{ml}$ for $0,3,6$, and 9 h. Beta Actin served as the loading control (D). Cell viability assay of Hela Parkin cells and Hela Parkin with USP30 overexpression cells after AO treatment with or without ST-539. Cells were treated with $\mathrm{AO}$ at $5 \mathrm{ug} / \mathrm{ml}$ w/o $10 \mathrm{ug} / \mathrm{ml} \mathrm{ST}-539$ for $24 \mathrm{~h}$ and then incubated with resazurin for $2 \mathrm{~h}$. Fluorescence was read using $544 \mathrm{~nm}$ excitation and $590 \mathrm{~nm}$ emission wavelength (E). Western blot analysis of mitophagy proteins and the pro-apoptosis signal in Hela Parkin cells and Hela Parkin with USP30 overexpression cells after the AO treatment w/o ST-539. Cells were treated with AO at $5 \mathrm{ug} / \mathrm{ml} \mathrm{w} / \mathrm{o} 10 \mathrm{ug} / \mathrm{ml} \mathrm{ST-539}$ for 0, 3, and $6 \mathrm{~h}$. Beta Actin served as the loading control (F). Cell viability assay of Hela ATG5 knockout cells, Hela ATG5 knockout Parkin cells, and Hela ATG5 knockout Parkin USP30 cells after AO treatment. Cells were treated with $\mathrm{AO}$ at $5 \mathrm{ug} / \mathrm{ml}$ for $24 \mathrm{~h}$ and then incubated with resazurin for $2 \mathrm{~h}$. Fluorescence was read using544 $\mathrm{nm}$ excitation and $590 \mathrm{~nm}$ emission wavelength.

\section{Parkin and USP30 Regulate AKT/mTOR Signaling and Cell Survival in Response to Mitochondrial Stress}

$\mathrm{AKT} / \mathrm{mTOR}$ signal functions as a master signal for biogenesis and cell survival (Xu et al., 2020; Manning and Toker, 2017). Previous studies have indicated that the AKT/mTOR signal responds to multiple cellular stressors to promote cell survival (de la Cruz López et al., 2019; Xu et al., 2020; Yang et al., 2017). To investigate how the AKT/mTOR signaling responds to mitochondrial stress, we analyzed the protein levels of AKT, mTOR, P70S6K, and 4EBP1 in three cell lines: wild-type Hela cells, Hela Parkin cells, and Hela Parkin USP30 cells, following AO treatment. Western blot results indicated that the AKT/ mTOR signaling increased throughout AO treatment, suggesting that AKT is activated and upregulated in response to mitochondrial stress (Figure 2, a), consistent with previous studies that indicate that mitochondrial stress activates the AKT survival pathway (Yang et al., 2017; Guha et al., 2010). In Hela Parkin cells, Akt and mTOR protein levels decreased significantly after $6 \mathrm{~h}$ of AO treatment (Figure 2A and Supplementary Figures 2A,B). The downstream signals of the AKT/mTOR pathway, 4EBP1 and P70S6K, were also downregulated (Supplementary Material 2C-E). These result suggest that Parkin may suppress AKT/mTOR signaling during mitophagy. USP30 overexpression significantly increased AKT's protein level and upregulated AKT/mTOR signals during mitophagy (Figure 2A and Supplementary Figures 2A-E). Moreover, the addition of ST-539, a USP30 inhibitor, resulted in decreased AKT protein levels during mitophagy (Figure 2B). To test if mitophagy activity contributes to the regulation of AKT protein levels and cell apoptosis, we utilized Chloroquine, a lysosomal inhibitor, to inhibit mitophagy (Redmann et al., 2017; Mauthe et al., 2018). We demonstraed that the AKT protein levels and cleaved 
A

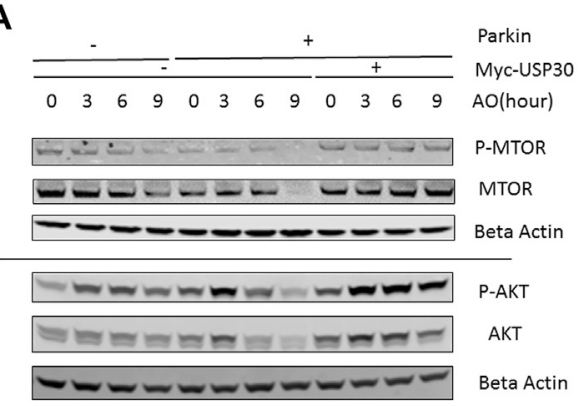

C

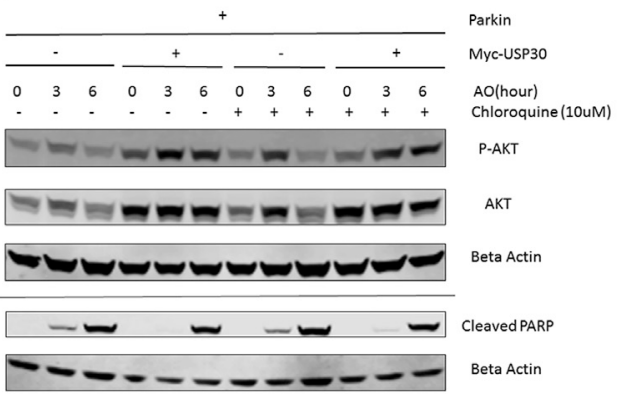

B

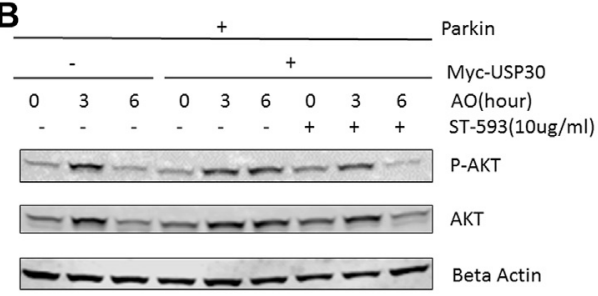

D

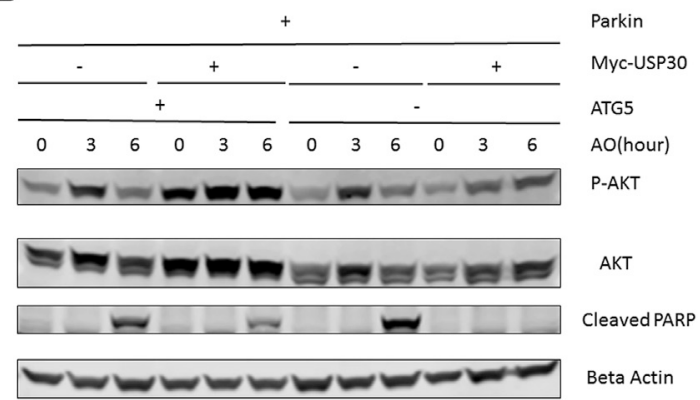

FIGURE 2 | USP30 upregulates AKT/mTOR signal (A). Western blot analysis of AKT/mTOR pathway proteins in Hela Parkin cells and Hela Parkin with USP30 overexpression cells after the AO treatment. Cells were treated with $\mathrm{AO}$ at $5 \mathrm{ug} / \mathrm{ml}$ for $0,3,6$, and $9 \mathrm{~h}$. Beta Actin served as the loading control. 2 (B). Western blot analysis of AKT signal in Hela Parkin cells and Hela Parkin with USP30 overexpression cells after the AO treatment w/o ST-539. Cells were treated with AO at 5 ug/ml w/o ST-539 for 0, 3, and 6 h. Beta Actin served as the loading control. 2 (C). Western blot analysis of AKT and cleaved PARP in Hela Parkin and Hela Parkin USP30 cells after the $\mathrm{AO}$ treatment $\mathrm{w} / \mathrm{o}$ chloroquine. Cells were treated with $\mathrm{AO}$ at $5 \mathrm{ug} / \mathrm{ml}$ for 0,3 , and $6 \mathrm{~h}$ with DMSO or $10 \mathrm{uM}$ chloroquine to inhibit autophagy/mitophagy. Beta Actin served as the loading control. 2 (D). Western blot analysis of AKT and cleaved PARP in Hela ATG5 knockout Parkin and Hela ATG5 knockout Parkin USP30 cells after the $\mathrm{AO}$ treatment. Cells were treated with $\mathrm{AO}$ at $5 \mathrm{ug} / \mathrm{ml}$ for 0,3 , and $6 \mathrm{~h}$. Beta Actin served as the loading control.
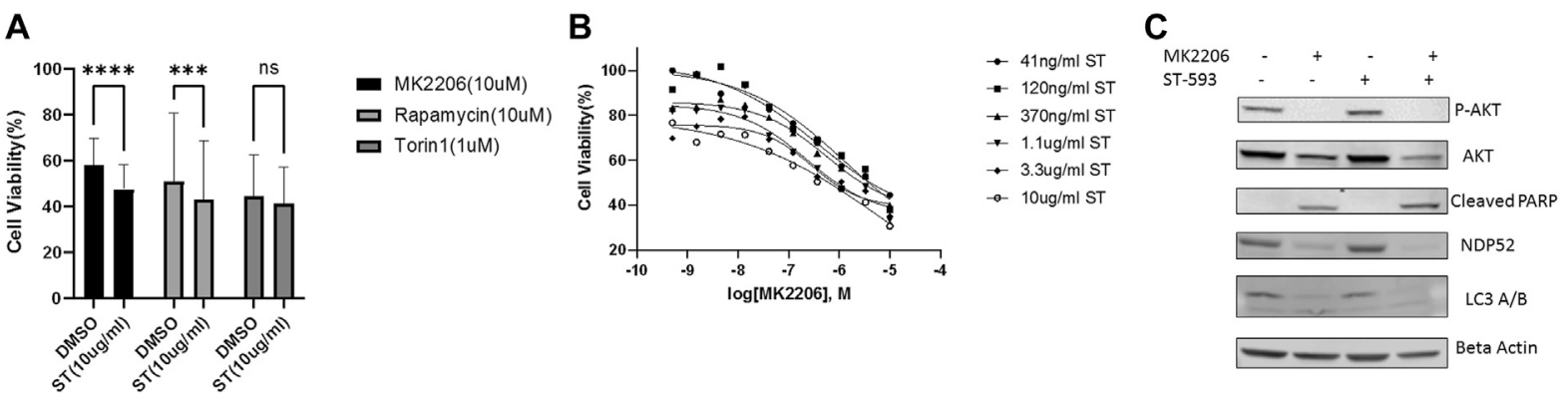

FIGURE 3 |USP30 in cancer treatment. 3 (A). Cell viability assay of Hela Parkin USP30 cells after AKT/mTOR inhibitors treatment w/o ST. Cells were treated with $10 \mathrm{uM}$ MK2206 or 10uM Rapamycin or $1 \mathrm{uM}$ Torin 1 for $48 \mathrm{~h}$ with DMSO or $10 \mathrm{ug} / \mathrm{ml} \mathrm{ST-539}$ and then incubated with resazurin for $2 \mathrm{~h}$. Fluorescence was read using $544 \mathrm{~nm}$ excitation and $590 \mathrm{~nm}$ emission wavelength. 3 (B). Cell viability assay on Jurkat T cells after $72 \mathrm{~h} \mathrm{MK2206}$ treatment with ST. Cells were treated with MK2206 and $\mathrm{ST}$ in the concentration gradient manner for $72 \mathrm{~h}$. After the treatment, cells were incubated with resazurin for $2 \mathrm{~h}$. Fluorescence was read using544 $\mathrm{nm}$ excitation and $590 \mathrm{~nm}$ emission wavelength. Each dot is the mean value of three biologically independent experiments. Trend lines are non-linear regression fitting curves. 3 (C). Western blot analysis of AKT, mitophagy, and pro-apoptosis signal in Jurkat T cells treated with DMSO, MK2206, or ST. Cells were treated with DMSO, MK2206, ST, or MK220 + ST for $24 \mathrm{~h}$. Beta Actin served as the loading control.

PARP following AO treatment were not affected after the addiotion of Chloroquine (Figure 2C). Similar results were observed in Hela Parkin and Hela Parkin USP30 cells that lack ATG5. (Figure 2D). These results suggest that AKT/mTOR signaling is activated in response to mitochondrial stress, and can be regulated by Parkin and USP30.

\section{USP30 May Serve as a Therapeutic Target for Leukemia Treatment}

We next asked whether USP30 could be a viable target to synergize with $\mathrm{AKT} / \mathrm{mTOR}$ inhibitors for leukemia treatment. We analyzed USP30's effect on AKT/mTOR inhibitors by measuring the viability of Hela Parkin USP30 cells after treatment with MK2206 (10uM), 
A

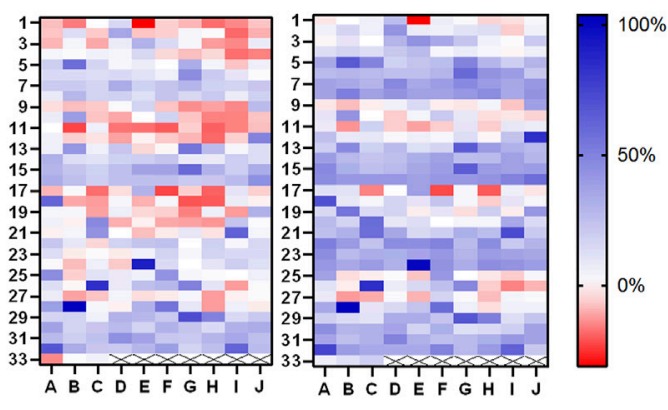

c

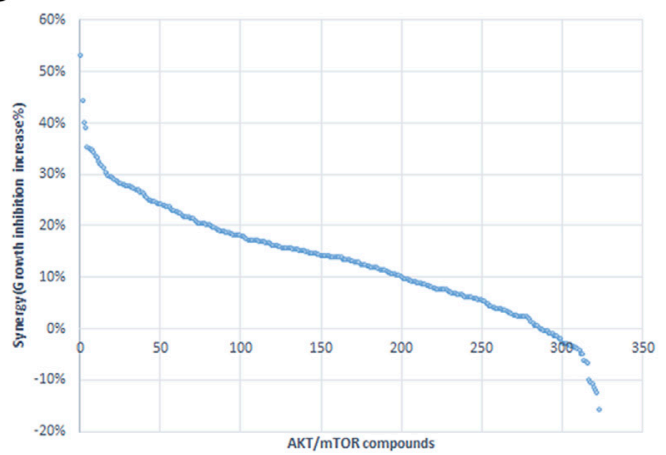

B

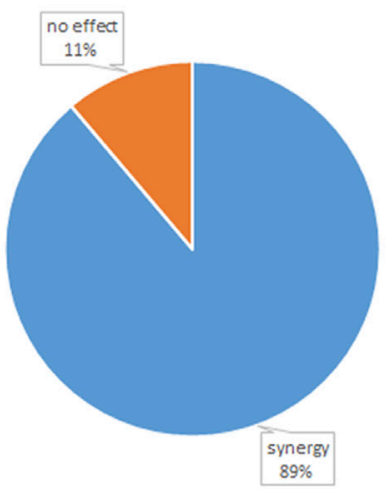

D

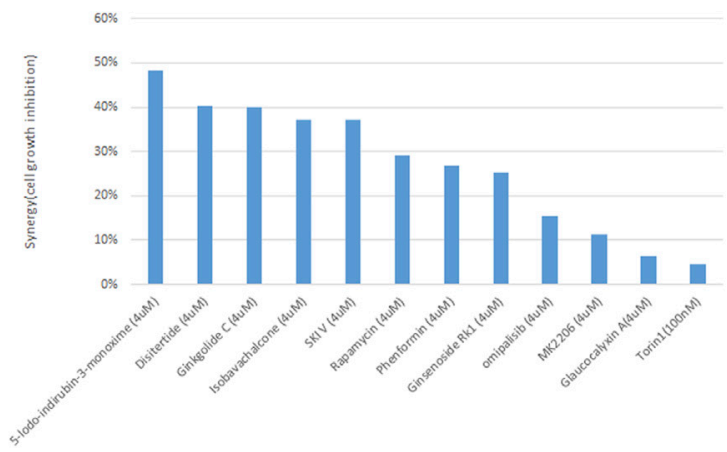

FIGURE 4 | Small targeted chemical screen using the PI3K/Akt/mTOR compound library. 4 (A). The heat map analysis of synergy between ST-539 and AKT/mTOR inhibitors on Jurkat cells growth inhibition. Jurkat T cells were treated with the compounds (4 uM) from PI3K/Akt/mTOR compound library (322 in total) w/o 10ug/ml ST539 for $48 \mathrm{~h}$, and the cell growth was analyzed by resazurin assay. The left one is the inhibition rate without ST-539, and the right one is the inhibition rate with ST-539. Data are the mean value from three biologically independent experiments. 4 (B). The percentage of the compounds from PI3K/Akt/mTOR compound library synergize with ST-539 on Jurkat cells growth inhibition. 4 (C). Ranks of synergistic effects between AKT/mTOR compounds and ST-539. Synergy is calculated by subtracting the growth inhibition caused by the compound alone from the growth inhibition caused by the compound and ST-539 together. 4 (D). The synergy between specific compounds with ST-539. Data are the mean values from three biologically independent experiments.

Rapamycin (10uM), or Tronil1 $(10 \mathrm{nM})$ in the presence and absence of ST-539. The cell viability analysis showed that inhibiting USP30 promoted drug-induced apoptosis significantly (Figure 3A). The apoptosis in treated cells suggests that USP30 inhibitors combined with $\mathrm{AKT} / \mathrm{mTOR}$ inhibitors might prove a benefit in treating leukemia. The next set of experiments focused on Jurkat cells. Jurkat cells are immortalized human $\mathrm{T}$ lymphocytes used to study acute T cell leukemia (Abraham and Weiss, 2004). We evaluated Jurkat cells viability after MK2206 and ST-539 treatment. Jurkat cell growth data revealed that the addition of ST-539 significantly improved MK2206s efficacy in inhibiting cell growth. (Figure 3B). Western blot results indicated that ST-539 synergized with MK2006 to inhibit AKT activity and increase proapoptotic signaling (cleaved PARP) in Jurkat cells (Figure 3C).

We employed Jurkat cells to investigate the synergistic effects between ST-539 and AKT inhibitors. We conducted a small-scale pilot chemical screen for Jurkat cell viability focusing on the AKT/ mTOR compound library composed of 322 compounds, in the presence and absence of ST-539. Interestingly, $89 \%$ of the compounds work in synergy with ST-539 to significantly suppress cell proliferation (Figures 4A,B). These results suggest a synergistic effect between ST-539 and AKT inhibitors. Combined treatment of these inhibitors provides a unique approach to treat
$\mathrm{T}$ cell leukemia. We ranked and reevaluated the most synergetic combinations from the previous experiment to find the most efficacious combinations (Figures 4C,D). The compound, 5-lodoindirubin-3-monoxime (Indirubin), worked best with ST-539, suppressing cell growth by $48 \%$ (Figure 4D). The dose-response curve of indirubin, Glaucocalyxin A, and MK2206 w/o ST-539 showed that ST-539 promoted efficacy (Figures 5A-C). In short, combining USP30 inhibitors with AKT/mTOR compounds in treating leukemia warrants further investigation.

\section{DISCUSSION}

Whether mitophagy promotes or works as an agonist to cancer development is unclear. Mitophagy is vital in rewiring metabolic pathways to support cancer cells' high energy demands (Chourasia et al., 2015; Vara-Perez et al., 2019). Previous studies induced cell apoptosis by knocking down USP30 potentiated BH3, ETC inhibitors (e.g., antimycin A, oligomycin), uncouplers (e.g., FCCP), subsequently boosting Parkin-dependent mitophagy. The increase in Parkin-dependent mitophagy indicates that USP30 could be a drug target in cancer treatment (Carroll et al., 2014; Liang et al., 2015). We have demonstrated that inhibiting USP30 boosts 

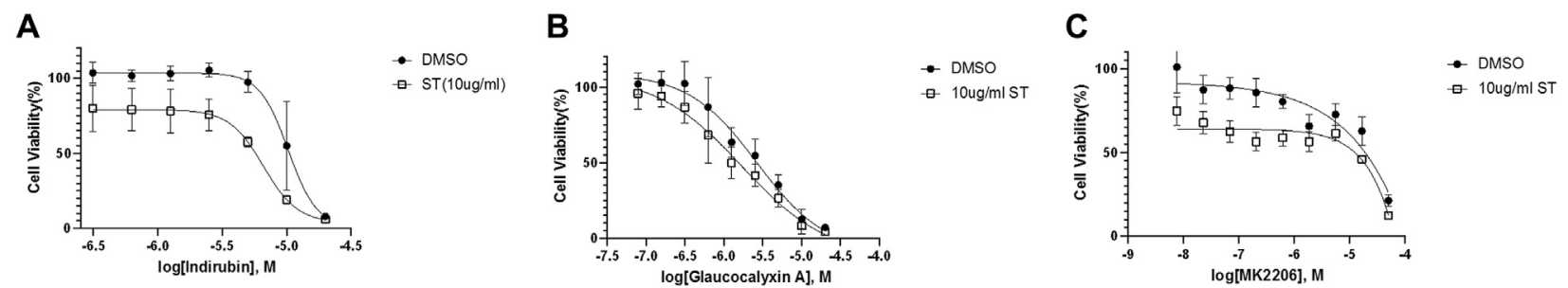

FIGURE 5| Selected inhibitors that show synergistic effects with ST-539 (A). The dose-response curve of 5-lodo-indirubin-3-monoxime on Jurkat T cells w/o ST539. Jurkat T cells were treated with 5-lodo-indirubin-3-monoxime in a concentration gradient manner with DMSO or 10 ug/ml ST-539 for $48 \mathrm{~h}$. The cell growth was analyzed using a resazurin assay (B). The dose-response of Glaucocalyxin A on Jurkat T cells w/o ST-539. Jurkat T cells were treated with Glaucocalyxin A in a concentration gradient manner with DMSO or $10 \mathrm{ug} / \mathrm{ml} \mathrm{ST-539}$ for $48 \mathrm{~h}$. The cell growth was analyzed using a resazurin assay (C). The dose-response of MK2206 on Jurkat T cells w/o ST-539. Jurkat T cells were treated with MK2206 in a concentration gradient manner with DMSO or 10 ug/ml ST-539 for 48 h. The cell growth was analyzed using a resazurin assay.

mitophagy and downregulates AKT signaling, promoting apoptosis during mitochondrial stress. Further drug screening using Jurkat cells demonstrates that the combination of USP30 inhibitors and AKT inhibitors is efficacious in treating $\mathrm{T}$ cell leukemia. However, whether Parkin and USP30 regulate AKT protein levels via modulating ubiquitination during mitochondrial stress remains to be investigated.

Overall, this research revealed the connections between Parkin, USP30, and AKT signals, proving that USP30 inhibitors may be effectivein leukemia treatment. Future studies could focus on investigating whether USP30 promotes AKT signaling and drug resistance in clinical status and whether this combined therapy works on patient-derived cancer cells and murine models.

\section{METHODS}

\section{Cell Culture and Reagents}

All HeLa cell lines (wild-type, HeLa Parkin, HeLa Parkin PINK1 KO, HeLa Parkin USP30, HeLa Parkin Myr-AKT, HeLa Parkin Myr-AKT K179M, HeLa Parkin AKT-T308A/S473A, HeLa Parkin AKTT308D/473D) were grown in Dulbecco's minimum essential medium (DMEM) with $10 \%$ fetal bovine serum (FBS) supplemented and $1 \%$ penicillin-streptomycin. HeLa Parkin and HeLa Parkin PINK1 KO cells were previously described (Lazarou et al., 2015). Hela Parkin USP30 was generated using lentiviral vectors of pLVX-Puro-Myc-USP30, obtained from Addgene (Sowa et al., 2009). Jurkat T cells were grown in Roswell Park Memorial Institute Medium (RPMI-1640) with 10\% fetal bovine serum (FBS) supplemented and $1 \%$ penicillin-streptomycin. For AO treatment, cells were incubated in a growth medium with $5 \mu \mathrm{M}$ oligomycin and $5 \mu \mathrm{M}$ antimycin A (details in figures). ST51000539 (ST-539) purchased from TimTec. Other chemicals were from SigmaAldrich (St. Louis).

\section{Western Blotting}

Cells were lysed in RIPA buffer $(50 \mathrm{mM}$ Tris- $\mathrm{HCl}$, at $\mathrm{pH} 8.0$; $150 \mathrm{mM} \quad \mathrm{NaCl} ; 1 \% \quad(\mathrm{vol} / \mathrm{vol})$ Nonidet P-40; $0.5 \%$ sodium deoxycholate, $0.1 \%$ SDS and protease inhibitor cocktail (Roche)) on ice. Primary antibodies used as described: USP30 (Sino Biological Inc., 14,548-RP01, 1:500); p-AKT (Cell Signaling Technology, 4060S,
1:1000); Parkin (Cell Signaling Technology, 4211S, 1:1000); AKT (Cell Signaling Technology, 4685S, 1:1000); Cleaved PARP (Cell Signaling Technology, 5625S, 1:1000); OPTN (Proteintech, 10837-IAP, 1:1000); p-mTOR (Cell Signaling Technology, 5536S, 1:1000); mTOR (Cell Signaling Technology, 2983S, 1:1000); TOM20 (Cell Signaling Technology, 42406S, 1:1000); NDP52 (Cell Signaling Technology, 60732S, 1:1000); p-P70S6K (Cell Signaling Technology, 9234P, 1:1000); P70S6K (Cell Signaling Technology, 9202S, 1:1000); LC3A/B (Cell Signaling Technology, 4108S, 1:1000); Beta-Actin (Cell Signaling Technology, 3700S, 1:1000). Secondary antibodies anti-rabbit (LI-COR, 926-32,211, 1:15 000) and antimouse (LI-COR, 926-68,072, 1:15 000) IgG were used to incubate membranes at room temperature for $1 \mathrm{~h}$. Images were captured using the Odyssey system (LI-Cor).

\section{Drug Screening}

Jurkat T cells were grown in 96-wells-plates with compounds from the PI3K/Akt/mTOR compound library bought from MedChemEXpress. Cells were treated with compounds at concentrations described in the figure for $48 \mathrm{~h}$. The growth inhibition on cells was analyzed using a resazurin cell viability assay.

\section{Cell Viability Assay}

Resazurin was bought from R\&D Systems (AR002). Resazurin was added at a volume equal to $10 \%$ of the cell culture volume, and cells were incubated for 1 to $2 \mathrm{~h}$ at $37^{\circ}$. Fluorescence of the cell culture medium was read using $544 \mathrm{~nm}$ excitation and $590 \mathrm{~nm}$ emission wavelength.

\section{DATA AVAILABILITY STATEMENT}

The original contributions presented in the study are included in the article/Supplementary Material further inquiries can be directed to the corresponding author.

\section{AUTHOR CONTRIBUTIONS}

$\mathrm{RZ}$ is the first author who proposed research, carried out the experiment, collected and analyzed the data. NS is RZ's main 
advisor and the lab PI. GX, YZ, and NS contributed to the conception and design of the study. SO contributed to experiments and data. HL and JK edited the draft. All authors contributed to manuscript revision, read, and approved the submitted version.

\section{FUNDING}

This work was supported by grants from the National Institutes for Health to NS. (HL135051 and HL160581).

\section{REFERENCES}

Abraham, R. T., and Weiss, A. (2004). Jurkat T Cells and Development of the T-Cell Receptor Signalling Paradigm. Nat. Rev. Immunol. 4 (4), 301-308. doi:10.1038/nri1330

Akundi, R. S., Zhi, L., and Büeler, H. (2012). PINK1 Enhances Insulin-like Growth Factor-1-dependent Akt Signaling and protection against Apoptosis. Neurobiol. Dis. 45 (1), 469-478. doi:10.1016/j.nbd.2011.08.034

Arafeh, R., and Samuels, Y. (2019). PIK3CA in Cancer: The Past 30 Years. Semin. Cancer Biol. 59, 36-49. doi:10.1016/j.semcancer.2019.02.002

Baudot, A. D., Haller, M., Mrschtik, M., Tait, S. W., and Ryan, K. M. (2015). Using Enhanced-Mitophagy to Measure Autophagic Flux. Methods 75, 105-111. doi:10.1016/j.ymeth.2014.11.014

Bingol, B., and Sheng, M. (2016). Mechanisms of Mitophagy: PINK1, Parkin, USP30 and beyond. Free Radic. Biol. Med. 100, 210-222. doi:10.1016/j. freeradbiomed.2016.04.015

Bingol, B., Tea, J. S., Phu, L., Reichelt, M., Bakalarski, C. E., Song, Q., et al. (2014). The Mitochondrial Deubiquitinase USP30 Opposes Parkin-Mediated Mitophagy. Nature 510 (7505), 370-375. doi:10.1038/nature13418

Carroll, R. G., Hollville, E., and Martin, S. J. (2014). Parkin Sensitizes toward Apoptosis Induced by Mitochondrial Depolarization through Promoting Degradation of Mcl-1. Cell Rep 9 (4), 1538-1553. doi:10.1016/j.celrep.2014. 10.046

Chourasia, A. H., Boland, M. L., and Macleod, K. F. (2015). Mitophagy and Cancer. Cancer Metab. 3, 4. doi:10.1186/s40170-015-0130-8

Chu, C. T. (2011). Diversity in the Regulation of Autophagy and Mitophagy: Lessons from Parkinson's Disease. Parkinsons Dis. 2011, 789431. doi:10.4061/ 2011/789431

D’Amours, D., Sallmann, F. R., Dixit, V. M., and Poirier, G. G. (2001). Gain-offunction of poly(ADP-Ribose) Polymerase-1 upon Cleavage by Apoptotic Proteases: Implications for Apoptosis. J. Cel Sci 114 (Pt 20), 3771-3778.

de la Cruz López, K. G., Toledo Guzmán, M. E., Sánchez, E. O., and García Carrancá, A. (2019). mTORC1 as a Regulator of Mitochondrial Functions and a Therapeutic Target in Cancer. Front. Oncol. 9, 1373. doi:10.3389/fonc.2019. 01373

Dikic, I., and Elazar, Z. (2018). Mechanism and Medical Implications of Mammalian Autophagy. Nat. Rev. Mol. Cel Biol 19 (6), 349-364. doi:10. 1038/s41580-018-0003-4

Ding, W. X., and Yin, X. M. (2012). Mitophagy: Mechanisms, Pathophysiological Roles, and Analysis. Biol. Chem. 393 (7), 547-564. doi:10.1515/hsz-2012-0119

Estruch, M., Reckzeh, K., Vittori, C., Centio, A., Ali, M., Engelhard, S., et al. (2021). Targeted Inhibition of Cooperative Mutation- and Therapy-Induced AKT Activation in AML Effectively Enhances Response to Chemotherapy. Leukemia 35, 2030-2042. doi:10.1038/s41375-020-01094-0

Fransecky, L., Mochmann, L. H., and Baldus, C. D. (2015). Outlook on PI3K/AKT/ mTOR Inhibition in Acute Leukemia. Mol. Cel Ther 3, 2. doi:10.1186/s40591015-0040-8

Ge, P., Dawson, V. L., and Dawson, T. M. (2020). PINK1 and Parkin Mitochondrial Quality Control: a Source of Regional Vulnerability in Parkinson's Disease. Mol. Neurodegener 15 (1), 20. doi:10.1186/s13024-020-00367-7

\section{ACKNOWLEDGMENTS}

We thank Dr. Richard Youle for generously providing us with the Hela Parkin cell lines. We thank Dr. Miyawaki for the original mt-Keima construct.

\section{SUPPLEMENTARY MATERIAL}

The Supplementary Material for this article can be found online at: https://www.frontiersin.org/articles/10.3389/fphar.2022.816551/ full\#supplementary-material

Gobeil, S., Boucher, C. C., Nadeau, D., and Poirier, G. G. (2001). Characterization of the Necrotic Cleavage of poly(ADP-Ribose) Polymerase (PARP-1): Implication of Lysosomal Proteases. Cell Death Differ 8 (6), 588-594. doi:10. 1038/sj.cdd.4400851

Guha, M., Fang, J. K., Monks, R., Birnbaum, M. J., and Avadhani, N. G. (2010). Activation of Akt Is Essential for the Propagation of Mitochondrial Respiratory Stress Signaling and Activation of the Transcriptional Coactivator Heterogeneous Ribonucleoprotein A2. Mol. Biol. Cel 21 (20), 3578-3589. doi:10.1091/mbc.E10-03-0192

Khandia, R., Dadar, M., Munjal, A., Dhama, K., Karthik, K., Tiwari, R., et al. (2019). A Comprehensive Review of Autophagy and its Various Roles in Infectious, Non-infectious, and Lifestyle Diseases: Current Knowledge and Prospects for Disease Prevention, Novel Drug Design, and Therapy. Cells 8 (7), 674. doi:10. 3390/cells8070674

Killackey, S. A., Philpott, D. J., and Girardin, S. E. (2020). Mitophagy Pathways in Health and Disease. J. Cel Biol 219 (11), e202004029. doi:10.1083/jcb. 202004029

Kim, Y. C., and Guan, K. L. (2015). mTOR: a Pharmacologic Target for Autophagy Regulation. J. Clin. Invest. 125 (1), 25-32. doi:10.1172/JCI73939

Kluge, A. F., Lagu, B. R., Maiti, P., Jaleel, M., Webb, M., Malhotra, J., et al. (2018). Novel Highly Selective Inhibitors of Ubiquitin Specific Protease 30 (USP30) Accelerate Mitophagy. Bioorg. Med. Chem. Lett. 28 (15), 2655-2659. doi:10. 1016/j.bmcl.2018.05.013

Krishnmurthy, R. G., and Shradgan, F. M. (2014). Commentary-1 (Research Highlights: USP30 Regulates the Mitophagy-Mediated Neuroprotection in Parkinson's Disease). CNS Neurol. Disord. Drug Targets 13 (10), 1633-1634. doi:10.2174/1871527314666141226122809

Lazarou, M., Sliter, D. A., Kane, L. A., Sarraf, S. A., Wang, C., Burman, J. L., et al. (2015). The Ubiquitin Kinase PINK1 Recruits Autophagy Receptors to Induce Mitophagy. Nature 524 (7565), 309-314. doi:10.1038/ nature 14893

Liang, J. R., Martinez, A., Lane, J. D., Mayor, U., Clague, M. J., and Urbé, S. (2015). USP30 Deubiquitylates Mitochondrial Parkin Substrates and Restricts Apoptotic Cell Death. EMBO Rep. 16 (5), 618-627. doi:10.15252/embr. 201439820

Luo, H., Krigman, J., Zhang, R., Yang, M., and Sun, N. (2021). Pharmacological Inhibition of USP30 Activates Tissue-specific Mitophagy. Acta Physiol. (Oxf) 232, e13666. doi:10.1111/apha.13666

Mai, S., Muster, B., Bereiter-Hahn, J., and Jendrach, M. (2012). Autophagy Proteins LC3B, ATG5 and ATG12 Participate in Quality Control after Mitochondrial Damage and Influence Lifespan. Autophagy 8 (1), 47-62. doi:10.4161/auto.8.1. 18174

Manning, B. D., and Toker, A. (2017). AKT/PKB Signaling: Navigating the Network. Cell 169 (3), 381-405. doi:10.1016/j.cell.2017.04.001

Mauthe, M., Orhon, I., Rocchi, C., Zhou, X., Luhr, M., Hijlkema, K. J., et al. (2018). Chloroquine Inhibits Autophagic Flux by Decreasing AutophagosomeLysosome Fusion. Autophagy 14 (8), 1435-1455. doi:10.1080/15548627.2018. 1474314

Narendra, D. P., Jin, S. M., Tanaka, A., Suen, D. F., Gautier, C. A., Shen, J., et al. (2010). PINK1 Is Selectively Stabilized on Impaired Mitochondria to Activate Parkin. Plos Biol. 8 (1), e1000298. doi:10.1371/journal.pbio.1000298 
Nepstad, I., Hatfield, K. J., Grønningsæter, I. S., and Reikvam, H. (2020). The PI3KAkt-mTOR Signaling Pathway in Human Acute Myeloid Leukemia (AML) Cells. Int. J. Mol. Sci. 21 (8), 2907. doi:10.3390/ijms21082907

Ordureau, A., Paulo, J. A., Zhang, W., Ahfeldt, T., Zhang, J., Cohn, E. F., et al. (2018). Dynamics of PARKIN-dependent Mitochondrial Ubiquitylation in Induced Neurons and Model Systems Revealed by Digital Snapshot Proteomics. Mol. Cel 70 (2), 211-e8. doi:10.1016/j.molcel.2018.03.012

Palikaras, K., Lionaki, E., and Tavernarakis, N. (2018). Mechanisms of Mitophagy in Cellular Homeostasis, Physiology and Pathology. Nat. Cel Biol 20 (9), 1013-1022. doi:10.1038/s41556-018-0176-2

Park, S., Chapuis, N., Tamburini, J., Bardet, V., Cornillet-Lefebvre, P., Willems, L., et al. (2010). Role of the PI3K/AKT and mTOR Signaling Pathways in Acute Myeloid Leukemia. Haematologica 95 (5), 819-828. doi:10.3324/haematol.2009.013797

Redmann, M., Benavides, G. A., Berryhill, T. F., Wani, W. Y., Ouyang, X., Johnson, M. S., et al. (2017). Inhibition of Autophagy with Bafilomycin and Chloroquine Decreases Mitochondrial Quality and Bioenergetic Function in Primary Neurons. Redox Biol. 11, 73-81. doi:10.1016/j.redox.2016.11.004

Saha, S., Panigrahi, D. P., Patil, S., and Bhutia, S. K. (2018). Autophagy in Health and Disease: A Comprehensive Review. Biomed. Pharmacother. 104, 485-495. doi:10.1016/j.biopha.2018.05.007

Soutar, M. P. M., Kempthorne, L., Miyakawa, S., Annuario, E., Melandri, D., Harley, J., et al. (2018). AKT Signalling Selectively Regulates PINK1 Mitophagy in SHSY5Y Cells and Human iPSC-Derived Neurons. Sci. Rep. 8 (1), 8855. doi:10.1038/s41598-018-26949-6

Sowa, M. E., Bennett, E. J., Gygi, S. P., and Harper, J. W. (2009). Defining the Human Deubiquitinating Enzyme Interaction Landscape. Cell 138 (2), 389-403. doi:10.1016/j.cell.2009.04.042

Vara-Perez, M., Felipe-Abrio, B., and Agostinis, P. (2019). Mitophagy in Cancer: A Tale of Adaptation. Cells 8 (5), 493. doi:10.3390/cells8050493

Vives-Bauza, C., Zhou, C., Huang, Y., Cui, M., de Vries, R. L., Kim, J., et al. (2010). PINK1-dependent Recruitment of Parkin to Mitochondria in Mitophagy. Proc. Natl. Acad. Sci. U S A. 107 (1), 378-383. doi:10.1073/pnas.0911187107

Wanderoy, S., Hees, J. T., Klesse, R., Edlich, F., and Harbauer, A. B. (2020). Kill One or Kill the many: Interplay between Mitophagy and Apoptosis. Biol. Chem. 402 (1), 73-88. doi:10.1515/hsz-2020-0231

Xu, F., Na, L., Li, Y., and Chen, L. (2020). Roles of the PI3K/AKT/mTOR Signalling Pathways in Neurodegenerative Diseases and Tumours. Cell Biosci 10, 54. doi:10.1186/s13578-020-00416-0
Yang, J. H., Siroky, M. B., Yalla, S. V., and Azadzoi, K. M. (2017). Mitochondrial Stress and Activation of PI3K and Akt Survival Pathway in Bladder Ischemia. Res. Rep. Urol. 9, 93-100. doi:10.2147/RRU.S132082

Ye, X., Zhou, X. J., and Zhang, H. (2018). Exploring the Role of Autophagy-Related Gene 5 (ATG5) Yields Important Insights into Autophagy in Autoimmune/ Autoinflammatory Diseases. Front. Immunol. 9, 2334. doi:10.3389/fimmu.2018. 02334

Yoshii, S. R., Kishi, C., Ishihara, N., and Mizushima, N. (2011). Parkin Mediates Proteasome-dependent Protein Degradation and Rupture of the Outer Mitochondrial Membrane. J. Biol. Chem. 286 (22), 19630-19640. doi:10. 1074/jbc.M110.209338

Youle, R. J., and Narendra, D. P. (2011). Mechanisms of Mitophagy. Nat. Rev. Mol. Cel Biol 12 (1), 9-14. doi:10.1038/nrm3028

Zachari, M., Gudmundsson, S. R., Li, Z., Manifava, M., Cugliandolo, F., Shah, R., et al. (2019). Selective Autophagy of Mitochondria on a UbiquitinEndoplasmic-Reticulum Platform. Dev. Cel 50 (5), 627-e5. doi:10.1016/j. devcel.2019.06.016

Zheng, W., Xie, W., Yin, D., Luo, R., Liu, M., and Guo, F. (2019). ATG5 and ATG7 Induced Autophagy Interplays with UPR via PERK Signaling. Cell Commun Signal 17 (1), 42. doi:10.1186/s12964-019-0353-3

Conflict of Interest: The authors declare that the research was conducted in the absence of any commercial or financial relationships that could be construed as a potential conflict of interest.

Publisher's Note: All claims expressed in this article are solely those of the authors and do not necessarily represent those of their affiliated organizations, or those of the publisher, the editors and the reviewers. Any product that may be evaluated in this article, or claim that may be made by its manufacturer, is not guaranteed or endorsed by the publisher.

Copyright $\odot 2022$ Zhang, Ozgen, Luo, Krigman, Zhao, Xin and Sun. This is an openaccess article distributed under the terms of the Creative Commons Attribution License (CC BY). The use, distribution or reproduction in other forums is permitted, provided the original author(s) and the copyright owner(s) are credited and that the original publication in this journal is cited, in accordance with accepted academic practice. No use, distribution or reproduction is permitted which does not comply with these terms. 